\title{
A Hybrid Cleaning Scheduling Framework for Operations and Maintenance of Photovoltaic Systems
}

\author{
Zhonghao Wang, Zhengguo Xu, Member, IEEE, Bin Liu, Member, IEEE, Yun Zhang, and Qinmin Yang, Senior \\ Member, IEEE
}

\begin{abstract}
Dust deposition on the surface of photovoltaic (PV) modules is a nonnegligible factor that reduces a PV system's efficiency and reliability. Cleaning can remove dust, and the effect of cleaning on PV performance resembles that of maintenance. In this article, we propose a hybrid cleaning scheduling policy with periodic planning and dynamic adjustment for refining the operations and maintenance of PV systems. Specifically, the periodic planning stage aims for medium-term scheduling while the dynamic adjustment stage is tailed for short-term fine-tuning. In the former stage, we show that when the number of cleaning actions is fixed, a periodic cleaning strategy is optimal. Moreover, we derive the optimality condition under which the optimal cleaning interval can be determined. In the latter stage, based on the determined cleaning interval, we dynamically adjust the cleaning schedule with the forecast of meteorological parameters, PV power generation, and dust deposition in order to further minimize economic losses. In addition, we take the forecasting uncertainty into account and propose a new custom parameter called risk-taking tendency (RTT) which is able to quantify the risk preference of decision makers and analyze its influence on the scheduling policy. A case study is provided to illustrate the proposed strategy.
\end{abstract}

Index Terms-photovoltaic systems, operations and maintenance, cleaning scheduling, periodic planning, dynamic adjustment

\section{NOMENCLATURE}

\section{Parameters of the power generation ${ }^{\mathrm{a}}$}

$p \quad$ ideal generated power

$h \quad$ total daytime hours

$e \quad$ electricity selling price

$d \quad$ dust accumulation degree (DAD)

$w_{0} \quad$ cleaning cost per square meter

Manuscript received ; revised ; accepted . This work was supported by the National Natural Science Foundation of China under Grants 61751307 and 61973269 and the National Key Research and Development Program of China under Grant 2019YFB1705502. (Corresponding author: Zhengguo Xu.)

$Z$. Wang is with the State Key Laboratory of Industrial Control Technology, College of Control Science and Engineering, Zhejiang University, Hangzhou, 310027, China, and also with the City University of Hong Kong Shenzhen Research Institute, Shenzhen, China. (e-mail: 11632028@zju.edu.cn)

$\mathrm{Z}$. Xu is with the State Key Laboratory of Industrial Control Technology, College of Control Science and Engineering, Zhejiang University, Hangzhou, 310027, China. (e-mail: xzg @zju.edu.cn)

B. Liu is with the Department of Management Science, University of Strathclyde, Glasgow, UK. (e-mail: b.liu@strath.ac.uk)

Y. Zhang is with the Shanghai Electric Distributed Energy Technology CO. LTD, Shanghai, China. (e-mail: yunzhang1985@163.com)

Q. Yang is with the State Key Laboratory of Industrial Control Technology, College of Control Science and Engineering, Zhejiang University, Hangzhou, 310027, China. (e-mail: qmyang@zju.edu.cn) area of the PV modules

\section{Symbols in the periodic planning stage}

$t \quad$ time index in the scheduling time range: $t \in$ $[0, T]$

c cleaning schedule: $\boldsymbol{c}=\left[c_{1}, c_{2}, \ldots, c_{I}\right]$, where $c_{i}$ is the $i$ th cleaning instant

$y \quad$ total economic losses during the scheduling time range

economic loss per unit time

the cleaning interval in medium-term schedule

Symbols in the dynamic adjustment stage

$n$

index of the day in the scheduling time range: $n \in\{1,2, \ldots, N\}$

index of adjustment cycles: $m \in\{1,2, \ldots, M\}$

index of the decision-making day: $k \in$ $\left[W_{m}^{d}, \overline{W_{m}^{d}}\right]$

index of the assumed cleaning date when scheduling for the future: $j \in\left[k+N_{l}, \overline{W_{m}^{a}}\right]$

predictable time length

lead time of cleaning

start day and end day of the adjustment window start day and end day of the decision-making window

$\boldsymbol{R} \quad$ applied cleaning schedule after dynamic adjustment: $\boldsymbol{R}=\left[r_{1}, r_{2}, \ldots, r_{M}\right]$, where $r_{m}$ is the $m$ th applied cleaning date

$r_{m}^{\prime} \quad$ planned cleaning date before adjustment of the $m$ th cycle

$\psi \quad$ daily mean economic loss

a Parameters with prime symbol (') represent forecasted values. Capital letters represent seasonal average values.

\section{INTRODUCTION}

In recent years, the shortage of traditional energy such as fossil fuel and natural gas has constantly been a public concern; climate change and air pollution caused by the consumption of traditional energy resources have also been a critical issue. As a result, renewable resources have gained increasing attentions [1]. In particular, solar energy is one of the most promising renewable resources due to its availability and cleanliness [2]. Photovoltaic (PV) power generation is a mainstream technology to convert solar energy to electricity. 
The reliability and efficiency of PV systems raise more and more attentions as it is seriously influenced by environmental conditions and meteorological parameters. Technologies to increase the reliability and efficiency of PV systems have attracted numerous research interests and been significantly improved [3]-[5].

The components responsible for energy conversion in PV systems are called PV modules. Shading is a major factor that causes degradation of a PV system's reliability and efficiency because it prevents solar radiations from reaching the PV modules [6]. In broad terms, there are two main sources of shading-barriers like trees and buildings, and dust deposition on the surface of PV modules. The former can be largely avoided by careful location selection and installation for PVs [7]. However, dust can scatter and absorb solar radiations and thus has a continual impact on PV power generation. A significant number of experiments have been conducted to investigate the influence of dust deposition on the conversion efficiency. It has been reported that the performance degradation of PV systems attributed to dust varies with location, environmental conditions, and exposure duration. In general, the impact of dust deposition on the power output of PV modules can account for up to $60-70 \%$ [8]-[10]. Such influence on the PV efficiency will, in turn, reduce the accuracy of PV output prediction, and therefore impedes an efficient and reliable energy management [11]-[13]. In addition, dust deposition may lead to hot spots on the surface of PV modules, which will reduce the efficiency and reliability of the system and even cause damage to the modules [14]-[16].

Although rain and wind can remove dust to some extent, dust deposition is inevitable for in-service PV systems. Cleaning as a special form of maintenance [17]-[22] is thus necessary to reduce the influence of dust deposition. To minimize the economic losses caused by dust deposition, an appropriate cleaning scheduling strategy should be developed to balance the efficiency restoration and the cleaning costs.

In practice, cleaning programs of PV systems should be designed by accounting for various factors including dust accumulation process, PV power generation, cleaning cost, etc. In the literature, however, limited studies have been devoted to the design of cleaning programs for PV systems. Pavan et al. [14] pointed out that the soiling influence is related to both the soil type and the cleaning technique. It was found that different cleaning schedules should be chosen according to these factors, though they did not provide methods to determine the schedule of cleaning. The optimal cleaning frequency was investigated in [23] by experiments on photovoltaic plants in Central Saudi Arabia. However, this scheme does not include the strategy of cleaning dates selection, and the assumption that the power generated during one time slot in the absence of soiling is a constant, is not realistic. Jiang et al. [24] found that in a desert area, modules should be cleaned when the power output reduction and the particle concentration are equal to $5 \%$ and $100 \mu \mathrm{g} / \mathrm{m}^{3}$, respectively. Al-Kouz et al. [25] investigated the effect of dust on a PV system built at Jordan and a recommendation for cleaning frequency of every two weeks was proposed. Zapata et al. [26] designed a cleaning program for a PV plant installed in northern Chile based on an analysis of energy losses. Hammad et al. [27] built two models, based on multivariate linear regression (MLR) and artificial neural network (ANN), to estimate PV system conversion efficiency using the experimental parameters of exposure time to natural dust and ambient temperature. Then they obtained an optimal cleaning frequency for a case study in Jordan based on the cost of cleaning and benefit of extra energy yields. Ashley et al. [28] theoretically analyzed the rationality of periodic cleaning for Concentrated Solar Power (CSP) systems. However, the dust accumulation process was assumed to be linear in time and the fluctuations caused by weather variations and other factors were ignored.

The majority of existing studies focused on a single PV system or plant and investigated dust disposition based on historical or experimental data. They focused on the PV system/plant itself rather than a general cleaning scheduling policy. Moreover, most existing studies did not consider daily variation of PV power generation. They implicitly assumed a periodic cleaning policy, and then determined an optimal cleaning interval, instead of a detailed cleaning schedule. $\mathrm{Ba}$ et al. [29] developed a condition-based cleaning strategy for PV systems with stochastic soiling, rain events, and imperfect cleanings. The optimal cleaning policy turns out to be a time-varying reflectivity threshold, below which cleaning is triggered. Wang et al. [30] proposed a dynamic scheduling strategy that considers daily variation of PV power generation with forecasting information. In their work, the dust accumulation process was oversimplified and failed to capture the real situations very well; also, their dynamic scheduling method depended heavily on the forecast, without fully utilizing static schedule derived from historical data.

This study aims to address the deficiencies above and fill the gaps in cleaning scheduling for PV systems. We develop a hybrid scheduling framework that integrates periodic planning and dynamic adjustment so as to minimize the economic losses due to dust deposition. The originality and main contributions are summarized as follows:

- Moving beyond solely considering the cleaning frequency, our method includes two stages - the periodic planning stage and the dynamic adjustment stage. The two stages can make full use of historical data, seasonal characteristic, and forecast of meteorological parameters and PV power generation to design a more refined cleaning schedule.

- In the periodic planning stage, rather than using a specific function of dust accumulation process, we consider a general function, and utilize seasonal characteristics of PV generation. We show that when the number of cleaning actions is fixed, a periodic cleaning policy is optimal; moreover, we derive the optimality condition under which the optimal cleaning interval can be determined.

- In the dynamic adjustment stage, we take the forecasting uncertainty into account and propose a new custom parameter called "risk-taking tendency" (RTT) to quantify the decision maker's risk attitude in the cleaning schedule.

The rest of this paper is organized as follows. Section II gives an overview of the research design and methodol- 


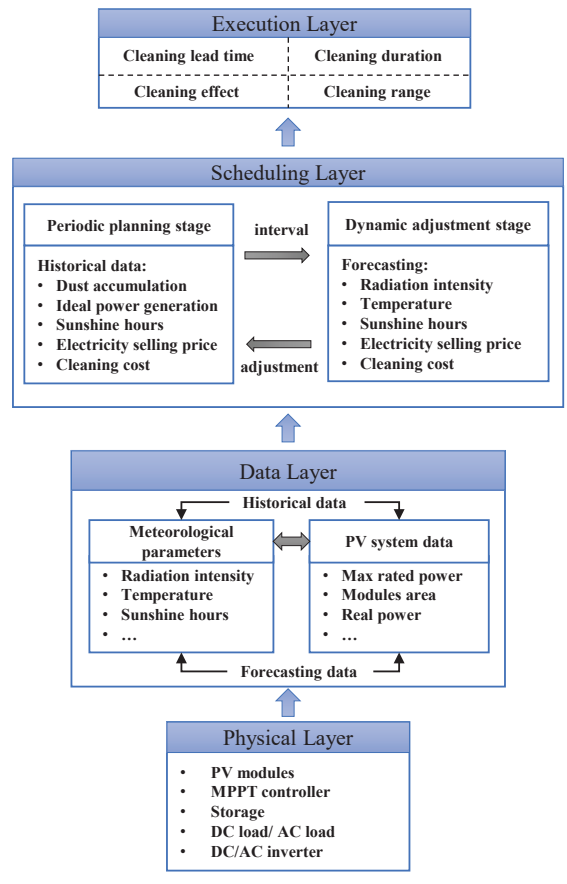

Fig. 1. Scheme of the hybrid scheduling framework with periodic planning and dynamic adjustment.

ogy. Section III formulates the cleaning scheduling problem and proposes the hybrid scheduling framework. Section IV presents a case study to illustrate the proposed cleaning framework, using real data from a PV system located in Shanghai, China. Section V concludes the paper.

\section{OVERVIEW OF THE FRAMEWORK}

With the aim of minimizing economic losses of PV systems, we propose a hybrid scheduling framework with two stages-the periodic planning stage and the dynamic adjustment stage. In the first stage we determine an optimal cleaning interval for the PV systems based on historical system characteristics and meteorological parameters. Unlike previous studies that focus only on a periodic cleaning schedule, in this study we further propose a dynamic adjustment policy under which the cleaning schedule in each cycle will be fine-tuned according to the forecast of meteorological parameters, PV power generation, and dust deposition. This implies that the cleaning interval determined in the periodic planning stage is just a rough schedule, and the actual interval between any two successive cleaning dates is adjusted by taking into account short-term variations. In essence, the adjustment in every cycle could improve the profit by considering specific condition variations, rather than only general conditions.

The proposed cleaning scheduling framework is illustrated in Fig. 1, which consists of four layers:

- Physical layer. This layer describes physical PV systems. A PV system consists of the PV modules, the Maximum Power Point Tracking (MPPT) controller, the energy storage part, the DC load/AC load, the DC/AC inverter, and other components. Some PV systems are connected to the power grid, called grid-connected systems, whereas others are named as off-grid PV systems. In this study, our focus is on the PV modules.

- Data layer. Data of meteorological parameters and PV systems are collected and transmitted to support the scheduling process. There are two types of data: historical data and forecasting data related to meteorological parameters and PV systems.

- Scheduling layer. This is the core layer to optimize the cleaning schedules. To minimize the total economic loss consists of power generation reduction caused by dust and cleaning cost, with the constraints of cleaning capacity, cleaning lead time and forecasting uncertainty, a two-stage cleaning scheduling policy is proposed. In the periodic planning stage, an optimal cleaning interval is determined based on the historical data as a preset schedule for the latter stage. In the dynamic adjustment stage, the cleaning schedule determined in the periodic stage might be advanced or postponed based on an adjustment algorithm to minimize the economic loss further. The scheduling problem and framework are detailed in Section III.

- Execution layer. The cleaning schedules are implemented in this layer. When applying the optimal schedule real systems, many factors should be taken into account. For example, the cleaning lead time indicates the lag from decision making to implementation, as it usually takes several days to prepare for cleaning. Cleaning duration, effect, and range are all factors that may vary with PV systems and situations.

\section{Hybrid Cleaning Scheduling Framework}

The hybrid cleaning scheduling framework is a two-stage process as previously mentioned. In the periodic planning stage, we show that when the number of cleaning actions is fixed, a periodic cleaning policy is optimal; moreover, we derive the optimality condition under which the optimal cleaning interval can be determined, so that the planning horizon of interest is divided into multiple non-overlapping cycles. In the dynamic adjustment stage, the cleaning schedule in each cycle determined in the former stage might be advanced or postponed based on an adjustment algorithm, and the decision maker's risk attitude is quantified and considered in the scheduling framework.

\section{A. Periodic planning stage}

Cleaning can remove dust deposition on the surface of PV modules to avoid the reduction of generation efficiency. If the PV modules are cleaned frequently, they can keep a high generation efficiency and thus gain more economic benefits. However, manual cleaning requires expenditure to pay for the water, detergent, labors, etc. Too frequent cleaning may result in an overwhelmed cost of cleaning against the benefit. On the other hand, the lack of cleaning may significantly affect the electricity generation and therefore reduce the profit. Therefore, one needs to optimize the cleaning interval to balance cleaning cost and benefit. 
In the cleaning-interval optimization problem, the dust accumulation process is a key element. In general, most existing studies just choose a specific prediction model to describe the dust accumulation process and then specify cleaning schedules accordingly. The dust accumulation process, however, varies with the PV systems, locations, seasons, and other factors. In the periodic planning stage, we propose a framework to determine an optimal cleaning interval for a general dust accumulation model. When applying to a specific PV system, the model can be fitted from the historical data available.

To quantify the effect of dust on power losses, we use the power loss ratio caused by dust, denoted by $d(t, \boldsymbol{c})$, to measure the dust accumulation degree (DAD) over an extend period from $t=0$ to $T$ when the cleaning schedule is $\boldsymbol{c}=\left[c_{1}, c_{2}, \ldots, c_{I}\right]$, where $d(t, \boldsymbol{c}) \in[0,1], t \in[0, T]$, $0=c_{0}<c_{1}<c_{2}<\cdots<c_{I}<T$ are cleaning implementation instants, and $I$ is the total number of cleaning times during $[0, T]$. Specifically, $d(t, c)$ consists of a deterministic term $f(t)$ and a random term $\Delta f(t)$, where $f(t)$ represents the underlying dust accumulation process of a PV system exposed to outdoor conditions without cleaning, and $\Delta f(t)$ is the random fluctuation caused by weather variations, air cleanliness, and other factors. In this study, $f(t)$ is modeled by a monotone increasing function and $\Delta f(t)$ by a Wiener process [31]-[33].

In this manner, the DAD $d(t, c)$ can be expressed as:

$$
\begin{aligned}
& d(t, c)=f\left(t-c_{i}\right)+\Delta f\left(t-c_{i}\right), \\
& t \in\left[c_{i}, c_{i+1}\right), i=0,1, \ldots, I, \\
& \Delta f(t) \sim \mathcal{N}\left(0, \sigma_{f}^{2} t\right),
\end{aligned}
$$

where $\sigma_{f}$ is the diffusion coefficient of the Wiener process. Here, the cleaning is regarded as a transient behavior and we assume that it is perfect, i.e., the dust deposition will be removed completely after each cleaning (will be explained later). Taking into account the generated power, the electricity selling price, and the cleaning cost, the total economic losses resulting from dust deposition and cleaning within $[0, T]$ can be expressed as:

$$
y(T, \boldsymbol{c})=\int_{0}^{T} p(t) d(t, \boldsymbol{c}) e(t) d t+I w_{0} S,
$$

where

- $p(t)$ is the ideal generated power (in $\mathrm{kW}$ ). Note that $p(t)$ is not necessarily the same as the rated maximum power of the PV system. "Ideal" here means that the modules are clean without any dust deposition. PV systems work at the rated maximum power only in the standard meteorological conditions. In practice, however, the actual radiation intensity and temperature are usually different from the standard conditions. Thus, the ideal generated power $p(t)$ is just the maximum power in certain meteorological conditions without the influence from dust. The actual generated power will be reduced by dust accumulation. The loss part caused by dust under cleaning schedule $c$ is $p(t) d(t, \boldsymbol{c})$.

- $e(t)$ is the electricity selling price (in currency unit/kWh). The power generation loss times the electricity selling price yields the economic loss resulting from dust deposition.

- $w_{0}$ and $S$ are the cleaning cost per square meter (in currency unit $/ \mathrm{m}^{2}$ ) and the cleaning area of the PV modules (in $\mathrm{m}^{2}$ ), respectively. In practice, a PV plant usually signs a one-year or longer outsourcing contract with a third-party company for cleaning operations. Within the contract period, the one-time cleaning cost per area of PV modules is usually a constant.

The theoretical global optimal cleaning schedule can be obtained if all the information about $p(t), e(t)$, and $d(t, \boldsymbol{c})$ in the future are exactly known. In reality, however, this is not the case. The ideal power generation depends heavily on the meteorological parameters. In general, the meteorological parameters for the same location are relatively stable in one season but may change across seasons. That is, $p(t)$ has a seasonal pattern. In the periodic planning stage, our aim is just to determine an optimal cleaning interval in an average manner. What we need is the average state of the PV power generation over an extended period. Thus, we can make full use of the seasonal patterns of PV generation. Moreover, according to the current practice, the electricity selling price is usually a constant within one year. Hence, for the time range in one season, we can approximate $p(t)$ and $e(t)$ by the corresponding seasonal average values $P$ and $E$, respectively, which can be obtained from the historical data. In that case, we can divide the whole scheduling horizon into several pieces by season and optimize the cleaning intervals for every season separately.

For convenience of description, the scheduling time range $T$ is assumed as one season in this article. A schedule for a period over several seasons or years can be connected by the specific schedule for each season. In addition, the generation efficiency of PV systems degrades over time inherently. Industrial standards and academic research show a median efficiency degradation of $0.5 \% / y e a r$ based on nearly 2000 case studies [34]-[36]. Such efficiency degradation rate is quite small so that it can be ignored in the cleaning scheduling problem. As a result, Eq. (2) can be rewritten as:

$$
y(T, \boldsymbol{c})=\int_{0}^{T} \operatorname{PEd}(t, \boldsymbol{c}) d t+I w_{0} S .
$$

The objective of cleaning scheduling is to determine an optimal cleaning interval so as to minimize the expected economic losses caused by the electricity reduction from dust as well as the cleaning cost over an extended period. Therefore, the optimization problem can be formulated as

\section{Problem 1}

$$
\begin{aligned}
& \min _{\boldsymbol{c}} \boldsymbol{E}[y(T, \boldsymbol{c})] \\
& \text { s.t. } 0<c_{i}<c_{j}<T, \forall 1 \leq i<j \leq I .
\end{aligned}
$$

For a fixed number of cleaning actions $I$, minimizing $\boldsymbol{E}[y(T, \boldsymbol{c})]$ is equivalent to minimizing $\boldsymbol{E}\left[\int_{0}^{T} P E d(t, \boldsymbol{c}) d t\right]$. We thus have the following result.

Theorem 1. When the number of cleaning actions is fixed, a periodic cleaning strategy is optimal. 
Proof. The term $\int_{0}^{T} P E d(t, c) d t$ can be expressed as

$$
\begin{aligned}
& \int_{0}^{T} P E d(t, c) d t \\
& =P E\left[\int_{0}^{c_{1}}(f(t)+\Delta f(t)) d t+\int_{c_{1}}^{c_{2}}\left(f\left(t-c_{1}\right)\right.\right. \\
& \left.\left.\quad+\Delta f\left(t-c_{1}\right)\right) d t+\cdots+\int_{c_{I}}^{T}\left(f\left(t-c_{I}\right)+\Delta f\left(t-c_{I}\right)\right) d t\right] \\
& =P E\left\{\left[\int_{0}^{c_{1}} f(t) d t+\int_{c_{1}}^{c_{2}} f\left(t-c_{1}\right) d t+\ldots\right.\right. \\
& \left.\quad+\int_{c_{I}}^{T} f\left(t-c_{I}\right) d t\right]+\left[\int_{0}^{c_{1}} \Delta f(t) d t+\int_{c_{1}}^{c_{2}} \Delta f\left(t-c_{1}\right) d t\right. \\
& \left.\left.+\ldots+\int_{c_{I}}^{T} \Delta f\left(t-c_{I}\right) d t\right]\right\} .
\end{aligned}
$$

The expectation of $\Delta f(t)$ for any $t \in[0, T]$ is zero, i.e., $\boldsymbol{E}[\Delta f(t)]=0, \forall t \in[0, T]$, due to the fact that $\Delta f(t)$ is a Wiener process. Thus, we have

$$
\begin{aligned}
\boldsymbol{E}\left[\int_{0}^{c_{1}} \Delta f(t) d t+\int_{c_{1}}^{c_{2}} \Delta f\left(t-c_{1}\right) d t\right. \\
\left.\quad+\cdots+\int_{c_{I}}^{T} \Delta f\left(t-c_{I}\right) d t\right] \\
=\int_{0}^{c_{1}} \boldsymbol{E}(\Delta f(t)) d t+\int_{c_{1}}^{c_{2}} \boldsymbol{E}\left(\Delta f\left(t-c_{1}\right)\right) d t \\
\quad+\cdots+\int_{c_{I}}^{T} \boldsymbol{E}\left(\Delta f\left(t-c_{I}\right)\right) d t \\
=0
\end{aligned}
$$

Therefore, the expectation of $\int_{0}^{T} P E d(t, c) d t$ is given by

$$
\begin{aligned}
\boldsymbol{E}\left[\int_{0}^{T} P E d(t, \boldsymbol{c}) d t\right]= & P E\left[\int_{0}^{c_{1}} f(t) d t+\int_{c_{1}}^{c_{2}} f\left(t-c_{1}\right) d t\right. \\
& \left.+\cdots+\int_{c_{I}}^{T} f\left(t-c_{I}\right) d t\right]
\end{aligned}
$$

Define $\widetilde{F}\left(c_{1}, c_{2}, \ldots, c_{I}\right)=\int_{0}^{c_{1}} f(t) d t+\int_{c_{1}}^{c_{2}} f\left(t-c_{1}\right) d t+$ $\cdots+\int_{c_{I}}^{T} f\left(t-c_{I}\right) d t$. As $f(t)$ is monotonically increasing in $t, \widetilde{F}(\cdot)$ is a convex function. Let $\widehat{y}(T, \boldsymbol{c})$ denote the total economic losses with a periodic cleaning strategy, then we have $\boldsymbol{E}[\widehat{y}(T, \boldsymbol{c})]=P E I \int_{0}^{\frac{T}{I}} f(t) d t+I w_{0} S$. Notice that $\widetilde{F}\left(c_{1}, c_{2}, \ldots, c_{I}\right) \geq I \int_{0}^{\frac{T}{I}} f(t) d t=\widetilde{F}\left(\frac{T}{I}, \frac{2 T}{I}, \ldots, \frac{I T}{I}\right)$ for all possible c. Therefore, we have $\boldsymbol{E}[y(T, \boldsymbol{c})]=$ $P E \widetilde{F}\left(c_{1}, c_{2}, \ldots, c_{I}\right)+I w_{0} S \geq P E I \int_{0}^{\frac{T}{T}} f(t) d t+I w_{0} S=$ $\boldsymbol{E}[\widehat{y}(T, \boldsymbol{c})]$ for all possible c, which implies that the best strategy is to adopt a periodic cleaning schedule.

The next problem is to find the optimal cleaning interval for the periodic cleaning strategy. To avoid the interference of $T$, we define the economic loss per unit time, $\eta(\alpha)$, as the objective function:

$$
\eta(\alpha)=\frac{\boldsymbol{E}[\widehat{y}(T, \boldsymbol{c})]}{T}=\frac{P E \int_{0}^{\alpha} f(t) d t+w_{0} S}{\alpha},
$$

where $\alpha$ is the cleaning interval.
In order to obtain the optimal cleaning interval $\alpha^{*}$, we take the first derivative of $\eta(\alpha)$ with respect to $\alpha$ :

$$
\eta^{\prime}(\alpha)=\frac{P E\left[f(\alpha) \alpha-\int_{0}^{\alpha} f(t) d t\right]-w_{0} S}{\alpha^{2}} .
$$

Setting $\eta^{\prime}(\alpha)=0$ yields

$$
f(\alpha) \alpha-\int_{0}^{\alpha} f(t) d t=\frac{w_{0} S}{P E} .
$$

Theorem 2. There exists a unique and finite solution $\alpha^{*}$ to (10), and the resultant economic loss per unit time is given by

$$
\eta\left(\alpha^{*}\right)=f\left(\alpha^{*}\right) \text {. }
$$

Proof. Define $\zeta(\alpha)=f(\alpha) \alpha-\int_{0}^{\alpha} f(t) d t$. Then, we have $\zeta^{\prime}(\alpha)=f^{\prime}(\alpha) \alpha \geq 0$. Notice that $\zeta(0)=0$ and $\zeta(\alpha) \rightarrow \infty$ as $\alpha \rightarrow \infty$. This implies that $\zeta(\alpha)$ is increasing in $\alpha$ from 0 to $\infty$. Therefore, the solution to (10) is unique and finite. Given the optimal solution $\alpha^{*}$ to (10), rearranging its terms yields (11).

Property 1. $\alpha^{*}$ and $\eta\left(\alpha^{*}\right)$ increase as $w_{0}$ and/or $S$ increases, while decrease as $P$ and/or $E$ increases.

As PV generation requires solar energy, PV systems only work during the daytime; they do not generate electricity at night. Cleaning is usually operated early in the morning near sunrise or in the evening near sunset to avoid influencing power generation. Therefore, the PV generation process can be regarded as a discrete process with one day as a time unit, and cleaning can be regarded as transient behavior. In practice, the aim of cleaning scheduling is to decide on the cleaning date, not the exact moment. Therefore, the optimization model can be transformed into a discrete problem. The period from $t=0$ to $T$ is divided into $N$ days, from day $n=0$ to day $N$. In this manner, the corresponding discrete form of (10) can be expressed as

$$
P E\left(f(\alpha) \alpha-\sum_{n=0}^{\alpha} f(n)\right)=w_{0} S,
$$

where the cleaning interval $\alpha$ is rounded to the nearest day.

Expression (12) can be well explained as follows. $f(\alpha)$ is the natural DAD up to $n=\alpha$. Hence, $\operatorname{PEf}(\alpha) \alpha$ is the economic loss with a fixed DAD $f(\alpha)$ of the system over $[0, \alpha] . P E \sum_{n=0}^{\alpha} f(n)$ represents the natural economic loss accumulated from 0 to $\alpha$ without cleaning. When the difference between $\operatorname{PEf}(\alpha) \alpha$ and $P E \sum_{n=0}^{\alpha} f(n)$ equals exactly to the one-time cleaning cost $w_{0} S$, the corresponding $\alpha$ is the optimal cleaning interval for this PV system. Because $f(t)$ is monotone increasing, we know that $f(\alpha) \alpha-\sum_{n=0}^{\alpha} f(n)$ is monotone increasing in $\alpha$. As a result, Property 1 well holds: The optimal cleaning interval $\alpha^{*}$ will become shorter if the ideal power generation and electric selling price increase. In this case, one should clean the PV system more frequently as the economic benefits of PV generation become larger. On the other hand, the optimal cleaning interval $\alpha^{*}$ will become longer if the cleaning cost increases and the dust deposition accumulates slower. Too frequent cleaning with high cleaning cost and slow dust deposition is unnecessary. When $\alpha^{*}>N$, no cleaning is needed. 


\section{B. Dynamic adjustment stage}

The optimal cleaning interval obtained from the previous stage is optimized in an average manner. In order to refine operations and maintenance, we propose a forecast-driven dynamic adjustment approach to further minimize the total economic losses. Every time upon the arrival of a planned cleaning date, we will set an adjustment window to consider if the cleaning date should be shifted to an earlier or later date. For example, if the optimal cleaning interval is one month and the length of the adjustment window is one week, then the adjustment will occur every one month before the planned cleaning date comes. The PV modules may be cleaned on the planned cleaning date, or several days earlier or later with a range of one week.

1) Adjustment process. The dynamic adjustment process for the whole scheduling horizon is illustrated in Fig. 2. To start with, we obtain the optimal cleaning interval $\alpha^{*}$ from the periodic planning stage. For example, let (1)-(4) denote four planned cleaning dates according to the planning schedule. Then, we specify an adjustment window for the first planned cleaning date. The actual implementation date of the first cleaning action (might be advanced or postponed) will be within this time window. However, the adjustment decision of the first cleaning date will be made a few days earlier because of the cleaning lead time. Usually, the cleaning manpower and resources need to be planned in advance, so we should start the decision making process before the adjustment window. To this end, we define a decision-making window which is a time range of the same length as the adjustment window but with a few days advanced. On the other hand, the decision making window will end the same time length earlier than the end of adjustment window. This is because decisions made after that day are too late from the cleaning preparation perspective. Suppose that the cleaning date in the first cycle is adjusted from (1) to (1) based on the adjustment algorithm, which will be introduced later. Then, the next planned cleaning date is $\alpha^{*}$ days later than (1), which means that (2)-(4) are all changed in accordance with the adjustment of the first cleaning date. By repeating the adjustment procedure, we can obtain the actual cleaning dates (1)-(4). It should be noted that the actual number of cleaning actions for the whole planning horizon may not necessarily be the same as that in the periodic planning schedule.

2) Adjustment window and decision-making window. Taking the $m$ th cycle as an example, the parameters for adjustment are shown in Fig. 3. The first step is to specify the adjustment window and the decision-making window. The length of both windows is set to $N_{f}-N_{l}$, where $N_{f}$ is the time period in which the meteorological and other parameters can be predicted based on current forecasting technologies, and $N_{l}$ is the lead time of cleaning, i.e., the shortest time period from cleaning planning to implementation. The adjustment window is specified in a manner that the next planned cleaning date is located in the midpoint of this window, and the decisionmaking window is $N_{l}$ days earlier than the adjustment window,

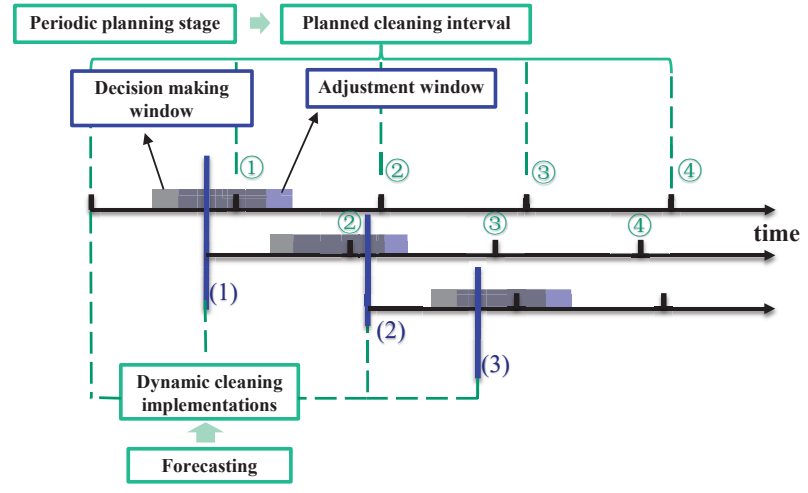

Fig. 2. Illustration of the adjustment process. In the periodic planning stage, we get (1)-(4) as planned cleaning dates. In the dynamic adjustment stage, before day (1), we set an adjustment window to choose an optimal cleaning date based on the forecasting data. Suppose that the executed cleaning date is advanced from (1) to (1). Then the subsequent planned cleaning dates (2)-(4) will also be advanced as the optimal cleaning interval is fixed. The executed dates in these cycles will be shifted to an earlier or later date in the same manner when their adjustment windows come.

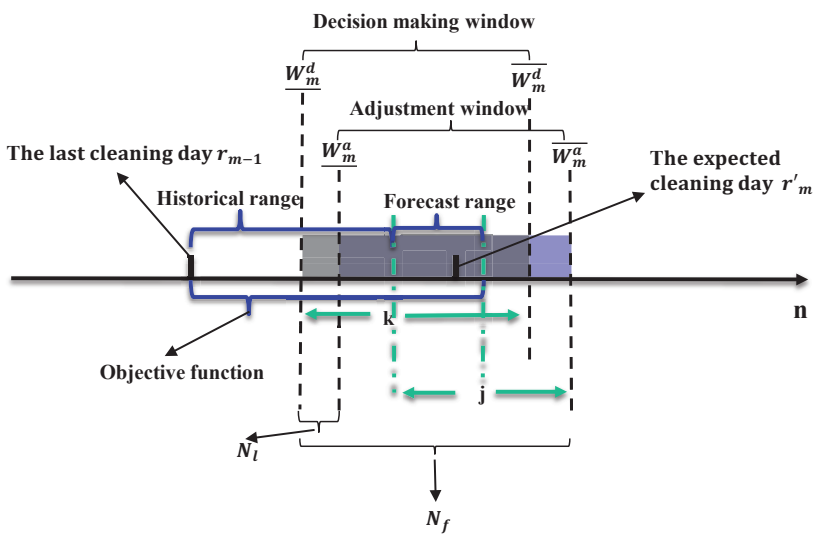

Fig. 3. Schematic illustration of the parameters for adjustment.

as shown in (13) and (14) below.

$$
\begin{aligned}
& {\left[\underline{W_{m}^{a}}, \overline{W_{m}^{a}}\right]=\left[r_{m-1}+\alpha^{*}-\frac{N_{f}-N_{l}}{2},\right.} \\
& \left.r_{m-1}+\alpha^{*}+\frac{N_{f}-N_{l}}{2}\right],
\end{aligned}
$$

and

$$
\begin{aligned}
& {\left[\underline{W_{m}^{d}}, \overline{W_{m}^{d}}\right]=\left[r_{m-1}+\alpha^{*}-\frac{N_{f}-N_{l}}{2}-N_{l},\right.} \\
& \left.r_{m-1}+\alpha^{*}+\frac{N_{f}-N_{l}}{2}-N_{l}\right],
\end{aligned}
$$

where $r_{m-1}$ is the actual cleaning date in the $(m-1)$ th cycle.

3) Decision algorithm. When the current date $k$ is $k=W_{m}^{d}$, the adjustment for the $m$ th cycle starts. We need to decide which day in $\left[k+N_{l}, \overline{W_{m}^{a}}\right]$ as the cleaning date. Let $\psi_{m, k}(j)$ represent the estimated daily economic loss from the last cleaning day to the cleaning date $j$ in this cycle. By evaluating 


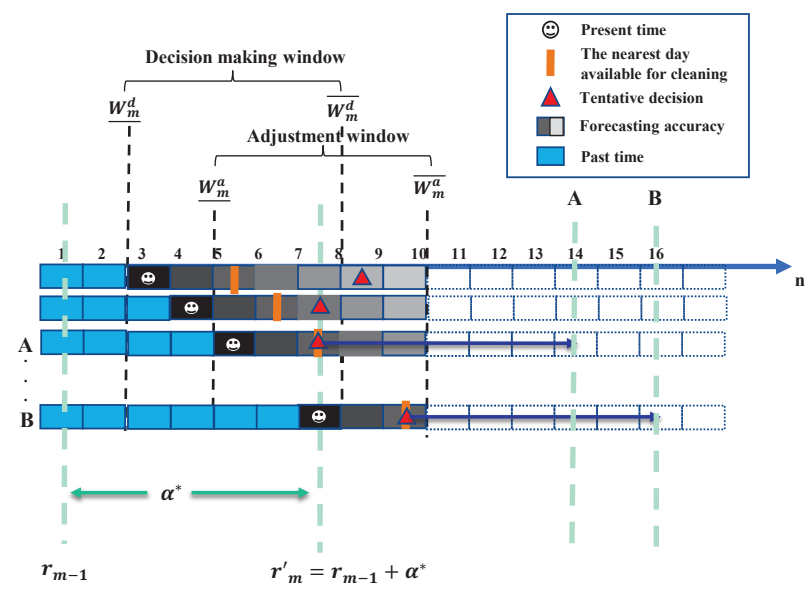

Fig. 4. An example of the adjustment decision making process for a single cycle. The diagrams from top to bottom illustrate the process over time. A and $\mathrm{B}$ are two kinds of final decisions. Each block represents one day. The forecasting accuracy is shown by the color shades of blocks.

$\psi_{m, k}(j)$ for every possible $j$, the "tentative cleaning date" $j_{m_{1}}$ of day $k$ in cycle $m$ can be determined as the date with the minimum $\psi_{m, k}(j)$, i.e., $j_{m_{1}}=\arg \min \boldsymbol{E}\left[\psi_{m, k}(j)\right]$.

$$
\begin{aligned}
\psi_{m, k}(j)= & \frac{\sum_{n=r_{m-1}}^{k} p_{n} h_{n} e_{n} d_{n}(\boldsymbol{R})+w_{0} S}{j-r_{m-1}+1} \\
& +\frac{\sum_{n=k+1}^{j} p_{n, k}^{\prime} h_{n, k}^{\prime} e_{n, k}^{\prime} d_{n, k}^{\prime}(j)}{j-r_{m-1}+1},
\end{aligned}
$$

where $p_{n}, h_{n}, e_{n}$ are the daily mean PV power, the total daytime hours, and electric selling price of day $n$, respectively. For period from $r_{m-1}$ to $k$, we have real data. For period from $k+1$ to $j$, we use notations with prime symbol (') to indicate that they are forecasting data. $\boldsymbol{R}=\left[r_{1}, r_{2}, \ldots, r_{m-1}\right]$ includes the dates when cleaning actions have been performed. $d_{n}(\boldsymbol{R}), n \in\left[r_{m-1}, k\right]$ are the DAD of the past days with cleaning schedule $\boldsymbol{R} \cdot d_{n, k}^{\prime}(j), n \in[k+1, j]$ are the forecasting DAD for the future days supposing $j$ is the cleaning date in this cycle.

The tentative cleaning date $j_{m_{1}}$ is the best choice when we making decision on day $k$, but it may not be executed because we can get more real data and more accurate forecasting information for the future over time. But if we are too conservative, we may miss the optimal decision. Thus, we have to design the execution conditions. When we get the tentative cleaning date $j_{m_{1}}$, if the execution conditions are met, the decision will be executed, then the adjustment for this cycle is completed; otherwise, we should wait for the next day, i.e. $k \leftarrow k+1$, and repeat the above process until $k=\overline{W_{m}^{d}}$.

4) Execution conditions. We determine whether or not to execute the tentative cleaning date according to the execution conditions. Fig. 4 illustrates the adjustment decision making process in a single cycle. Each block represents one day. The forecasting accuracy is shown by the color shades of blocks. As time comes closer, more historical data can be collected and more accurate forecast for the future days can be obtained. The updated information may result in a better cleaning schedule. Specifically, there are four parameters in Eq. (15) to be predicted. Among them, the electricity selling price is usually fixed in each year. The total daytime hours in each day is relatively stable in a single season so that the fluctuation can be ignored. We can use the seasonal mean values of the two parameters, $E$ and $H$ to replace their daily values. The function of DAD can be fitted from historical data.

Now the key parameter left is the ideal generated power. In the literature, most power forecasting technologies are statistical- or machine-learning-based methods, and can generate a probability distribution of the power [37]-[39]. Since the forecasting method is not the focus of this research, we directly use the forecast information as given. The predicted ideal generated power is modeled as a normally distributed random variable, as follows:

$$
p_{n, k}^{\prime} \sim \mathcal{N}\left(\mu_{p_{n, k}^{\prime}}, \sigma_{p_{n, k}^{\prime}}^{2}\right) .
$$

As a consequence, $\psi_{m, k}(j)$ is also a normally distributed random variable, as follows:

$$
\psi_{m, k}(j) \sim \mathcal{N}\left(\mu_{\psi_{m}(k, j)}, \sigma_{\psi_{m}(k, j)}^{2}\right),
$$

where

$$
\begin{aligned}
\mu_{\psi_{m, k}(j)}= & \frac{w_{0} S}{j-r_{m-1}+1}+\frac{H E}{j-r_{m-1}+1} \\
& \times\left(\sum_{n=r_{m-1}}^{k} p_{n} d_{n}(\boldsymbol{R})+\sum_{n=k}^{j} \mu_{p_{n, k}^{\prime}} d_{n, k}^{\prime}(j)\right),
\end{aligned}
$$

and

$$
\sigma_{\psi_{m, k}(j)}^{2}=\left(\frac{H E}{j-r_{m-1}+1}\right)^{2} \sum_{n=k}^{j} d_{n, k}^{2}(j) \sigma_{p_{n, k}^{\prime}}^{2} .
$$

We define the "nearest day available for cleaning" as the earliest day that can be booked for cleaning under the lead time constraints. On the $k$ th day, the nearest day available for cleaning is day $k+N_{l}$. If $j_{m_{1}}>k+N_{l}$, then the tentative decision will not be applied and the decision maker can update forecasts and thus decisions without missing $j_{m_{1}}$. If $j_{m_{1}}=k+N_{l}$, then the cleaning decision should be made by combining the decision maker's RTT. This is because applying the tentative decision $j_{m_{1}}$ means that the decision maker gives up the chance of getting a better schedule in this time window. RTT offers a useful tool to quantify such risk.

Let $j_{m_{2}}$ denote the cleaning date corresponding to the second minimum $\psi_{m, k}(j)$. Then the difference between $\psi_{m, k}\left(j_{m_{1}}\right)$ and $\psi_{m, k}\left(j_{m_{2}}\right)$, i.e., $\Delta \psi\left(m_{1}, m_{2}\right)$, is also a normally distributed random variable, namely, $\Delta \psi\left(m_{1}, m_{2}\right) \sim$ $\mathcal{N}\left(\mu_{\psi_{m, k}\left(j_{m_{1}}\right)}-\mu_{\psi_{m, k}\left(j_{m_{2}}\right)}, \sigma_{\psi_{m, k}\left(j_{m_{1}}\right)}^{2}+\sigma_{\psi_{m, k}\left(j_{m_{2}}\right)}^{2}\right)$. RTT is defined as the lowest probability of $\Delta \psi\left(m_{1}, m_{2}\right) \leq 0$ that the decision maker can accept. Because $\mu_{\psi_{m, k}\left(j_{m_{1}}\right)} \leq \mu_{\psi_{m, k}\left(j_{m_{2}}\right)}$, we have $\operatorname{Pr}\left\{\Delta \psi\left(m_{1}, m_{2}\right) \leq 0\right\} \in[0.5,1)$ and thus $R T T \in$ $[0.5,1)$. A higher RTT means a higher risk preference, which implies that the decision maker is more likely to take the risk to wait for a better chance. When $j_{m_{1}}=k+N_{l}$ and $\operatorname{Pr}\left\{\Delta \psi\left(m_{1}, m_{2}\right) \leq 0\right\}>R T T$, the tentative decision will be applied. If the condition is not satisfied until $k=\overline{W_{m}^{d}}$-the 
last day of the decision-making window, then there would be only one day to be selected as the cleaning day, i.e., $\overline{W_{m}^{a}}$.

In Fig. 4, we set $N_{f}=7, N_{l}=2, r_{m-1}=1$, and $\alpha^{*}=6$. On the 3rd and 4th days, the tentative cleaning days are both not the nearest days available for cleaning for these two days. On the 5 th day, the tentative date $j_{m_{1}}$ and the nearest day available for cleaning $k+N_{l}$ are both day 7, so the decision maker could decide on whether to perform cleaning or not according to the condition $\operatorname{Pr}\left(\Delta \psi\left(m_{1}, m_{2}\right) \leq 0\right)>R T T$. If the 7 th day is selected as the cleaning implementation day, then set $r_{m}=7$ and the scheduling for this cycle is completed. However, if the condition above is not satisfied until day 7 , then the last day of the adjustment window, i.e., day 9, would be the cleaning day. The dynamic adjustment algorithm is detailed in Algorithm 1. The convergence of this algorithm is guaranteed based on the increase of forecasting accuracy with a closer time distance. The decision maker can obtain more real data and the forecasting for the future will be more accurate over time. Thus, the results converge to the decision knowing all the real data with the decrease of uncertainty. For a scheduling time range with length of $n$, the computation complexity of Algorithm 1 is $\mathcal{O}(n)$, as the calculation times in one adjustment window is a constant independent of $n$ and the number of adjustment windows is $\frac{n}{\alpha^{*}}$.

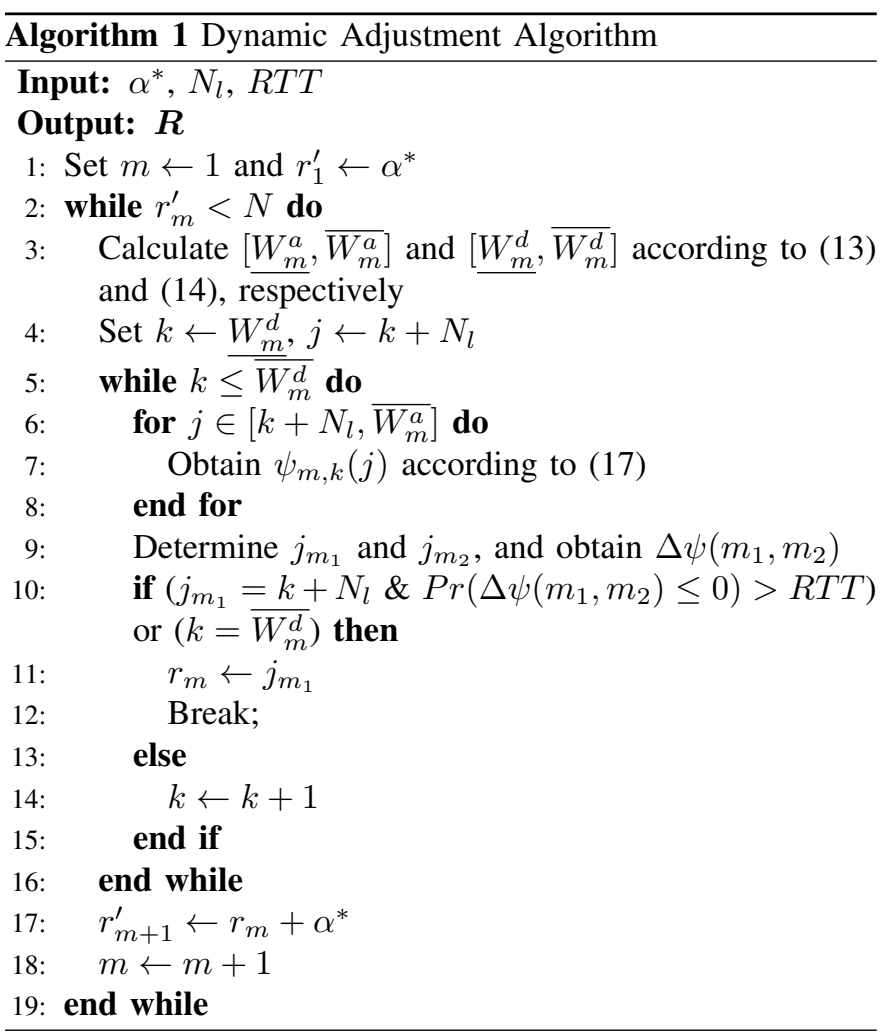

\section{CAse Study}

\section{A. Data description}

We collect data from a PV system located in Shanghai, China. The nominal values of the system parameters are listed in Table I, where STC stands for the "Standard Test Conditions". The environment measuring instruments and inverter
TABLE I

NOMINAL VALUES OF SYSTEM PARAMETERS.

\begin{tabular}{lll}
\hline Variable & Parameter & Value \\
\hline$P_{\text {max }}$ & Rated max power per module & $225 \mathrm{~W}$ \\
$N_{\text {penal }}$ & Number of modules & 18 \\
$S$ & Total area of the modules & $30 \mathrm{~m}^{2}$ \\
$G_{S T C}$ & Radiation of STC & $1000 \mathrm{~W} / \mathrm{m}^{2}$ \\
$T_{S T C}$ & Temperature of STC & $25^{\circ} \mathrm{C}$ \\
NOTC & Normal operating cell temperature & $45^{\circ} \mathrm{C}$ \\
\hline
\end{tabular}

monitoring instruments have recorded data since September 2015, and the sampling period is five minutes.

\section{B. Monte Carlo simulations}

The data of the system and meteorology in year 2016 are extracted for Monte Carlo simulations. We compare the yearly and seasonal economic losses of four scheduling methods to show the performance of our proposed method.

- Policy I: No cleaning.

- Policy II: Online periodic cleaning, which is designed by the periodic planning stage.

- Policy III: Offline periodic cleaning, provided that all the data is known. The result of this method is the best one that the periodic cleaning strategy can achieve.

- Policy IV: The proposed hybrid scheduling policy with periodic planning and dynamic adjustment.

In this case study, we adopt a widely adopted dust deposition process function to model the efficiency reduction due to dust [40]-[42]:

$$
f(t)=\kappa\left(1-e^{-\tau t}\right)+\Delta f(t) .
$$

One can see that $f(t)$ is an asymptotic curve, where $\kappa$ is the asymptotic value, $\tau$ is the accumulation rate factor, and $\Delta f(t)$ is the random fluctuation. In the simulation, the parameters are fitted season by season from the historical data.

We first compare the performances of the four methods under cleaning cost variation. The electricity selling price is set as $E=0.65 \mathrm{RMB} / \mathrm{kWh}$, according to current policy. The predictable time length is set as $N_{f}=7$ days, according to the existing power forecasting studies [37], [43]. The lead time of cleaning is set as $N_{l}=1$ day. We specify the RTT and forecasting accuracy as random values in order to demonstrate the performance of our method in different situations. According to the market research, the one-time cleaning cost per square meter ranges from 0.5 to $1 \mathrm{RMB} / \mathrm{m}^{2}$. Thus, we set three cost levels-0.5, 0.75, $1 \mathrm{RMB} / \mathrm{m}^{2}$, to perform simulations with 500 times, respectively.

Table II shows the average cleaning times under the hybrid scheduling policy with the three cleaning cost levels in each season as well as the whole year. One can observe that the PV system should be cleaned more frequently in spring and winter than in summer and autumn. This result coincides with the fact that the dust in the atmosphere is more serious in spring and winter, and there is more rain in summer and autumn in Shanghai. Obviously, the number of cleaning times in every season becomes smaller as the cleaning cost increases. When 
TABLE II

CLEANING TIMES UNDER TWO STAGE SCHEDULING METHOD IN EACH SEASON WITH CLEANING COST VARIATION.

\begin{tabular}{llll}
\hline Time Cost & & & \\
& 0.5 & 0.75 & 1 \\
\hline Season & & & \\
\hline Spring & 10.2 & 7.1 & 6.0 \\
Summer & 3.0 & 2.0 & 0.0 \\
Autumn & 3.0 & 2.0 & 1.0 \\
Winter & 6.0 & 5.0 & 4.0 \\
Whole year & 22.2 & 16.1 & 11.0 \\
\hline
\end{tabular}

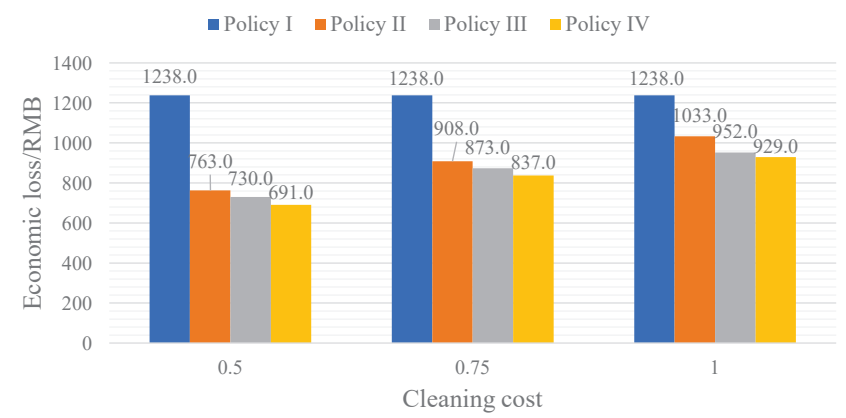

Fig. 5. Average whole year economic loss with the four methods.

the cleaning cost increases to $1 \mathrm{RMB} / \mathrm{m}^{2}$, there should be no cleaning in summer because the benefit from cleaning cannot offset the cost incurred.

Figs. 5 and 6 show the comparison results of the four methods. In Fig. 5, one can see that the economic loss can be significantly reduced when the system is cleaned, no matter which scheduling method is used. However, the economic losses of all the three cleaning policies will increase when the cleaning cost becomes higher. The proposed hybrid scheduling policy always has the best performance under all the three levels of cleaning cost. Fig. 6 further shows the average loss of all cost levels by seasons. Cleaning can cut down the economic loss in every season, and the performance of the hybrid scheduling policy is the best or tied for the best of all methods in all seasons; the superiority is more significant in spring and winter, because the efficiency reduction caused by dust is more serious in these two seasons. The performance comparison of the three scheduling policies is more clear in

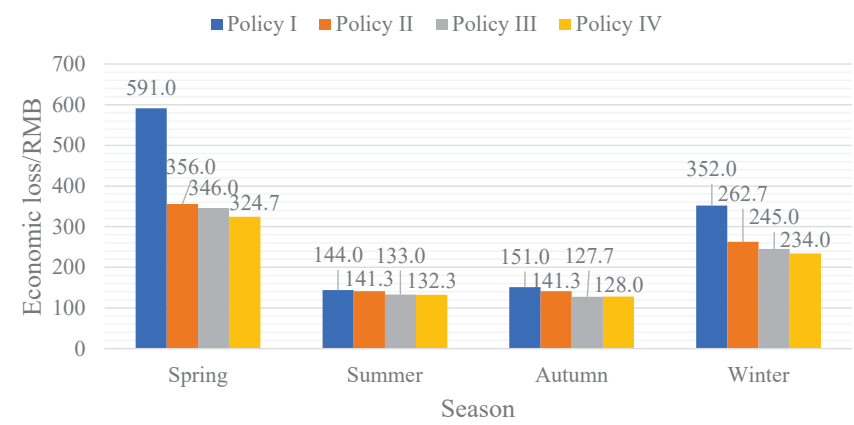

Fig. 6. Average seasonal economic loss with the four methods.

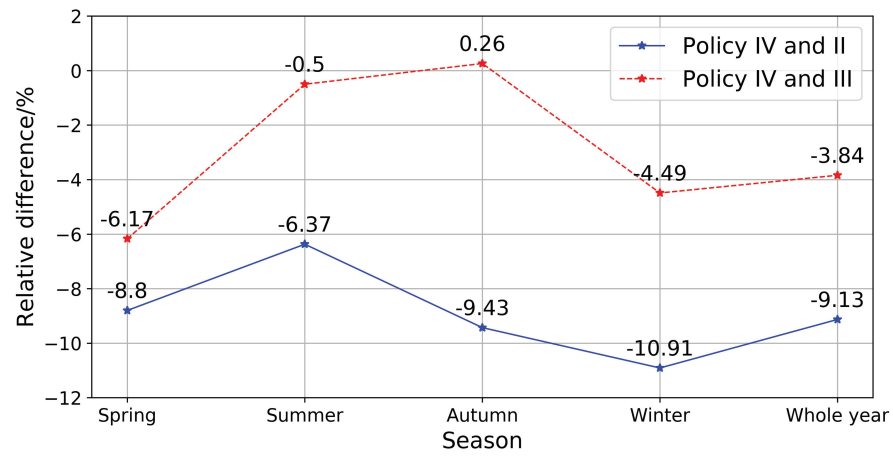

Fig. 7. Relative difference of the two stage scheduling and periodical cleaning.

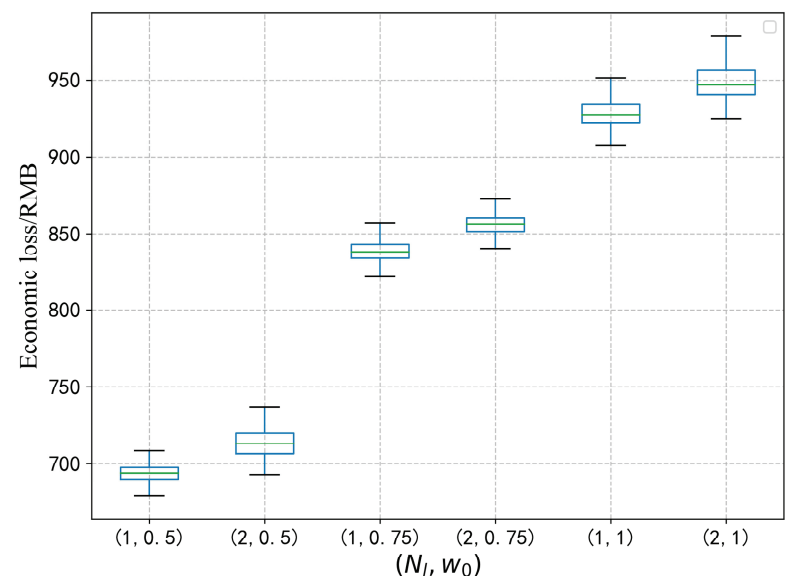

Fig. 8. Economic loss with cleaning lead time variation.

Fig. 7. Define the relative difference between the results of the policies as: Relative difference between Policy IV and Policy II (III) $=($ Results of Policy IV - Results of Policy II (III)) / Results of Policy II (III). One can see that the relative difference between the hybrid scheduling policy and the two periodic methods varies across seasons. The hybrid scheduling policy can bring a 6-11\% improvement in comparison with the online periodic cleaning policy. For the whole year, the average improvement is $9.13 \%$. Comparing with the offline periodic cleaning policy, the hybrid scheduling policy cannot make remarkable improvement in summer and even performs worse in autumn. The reason is that in summer and autumn the clean times are limited and the weather fluctuation is more volatile. The influence of dynamic adjustment is limited compared to the offline method with all data. For the whole year, however, the hybrid scheduling policy can reduce the economic loss by at least $3.84 \%$ when compared with the offline periodic cleaning policy.

1) Scheduling performance with lead time constraint variation: Lead time of cleaning refers to the shortest time period between cleaning planning and implementation. The 


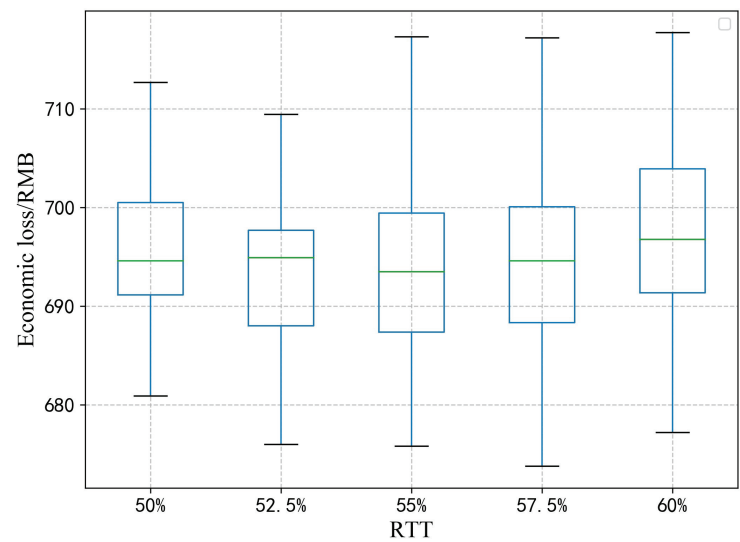

Fig. 9. Economic loss with RTT variation.

lead time constraint can be relaxed if the dispatch of cleaning manpower and resources are flexible, corresponding to a small $N_{l}$. The performances of the proposed method with lead time constraints under the three levels of cleaning cost are shown in Fig. 8. The box-plots include the upper bound, upper quartile, median, lower quartile, and lower bound of all the simulations to show the distribution of the results. From Fig. 8 one can see that the overall results increase with cleaning cost, and the distribution of results with $N_{l}=1$ is better than that with $N_{l}=2$ under every level of cost. This indicates that the proposed method performs better when the cleaning workers and resources are more abundant.

2) Scheduling performance with RTT variation: Risktaking tendency is a parameter decided by the decision maker. The influence of the risk preference to the results is shown in Fig. 9. $R T T=50 \%$ means that the decision maker do not consider the distribution of the forecasting. They make decision solely according to the expectation value optimization. Thus, the proposition of RTT can increase the performance of the cleaning scheduling. But it is not rational to choose an exorbitant RTT. A higher RTT means the decision maker prefers to wait for a better chance. They may miss the reasonable cleaning choices until they do not have chance to make scheduling. Fig. 9 shows the distributions of the results with RTT varying from 50-60\%. The medians of them are similar, but the distributions are different. For RTT equals to $52.5 \%, 55 \%$, and $57.5 \%$, the distribution range is larger gradually, which means that when the RTT is higher, the worst case may be worse, meanwhile the best case may be better. The decision maker have a chance to reduce more economic loss but also have the risk to loss more.

\section{CONCLUSIONS}

This study investigated the design of a hybrid cleaning scheduling policy for PV systems that integrates a periodic planning stage and a dynamic adjustment stage. The periodic planning stage decides on an optimal cleaning interval based on historical data. In this stage, we have shown that when the number of cleaning actions is fixed, a periodic cleaning strategy is optimal; moreover, we derived the optimality condition under which the optimal cleaning interval can be determined. The dynamic adjustment stage dynamically finetunes the cleaning date based on the optimized cleaning interval and the forecast of meteorological parameters, PV power generation, and dust deposition in order to further minimize the economic losses. The forecasting uncertainty is also considered. Furthermore, the adjustment algorithm incorporates RTT of the decision maker and evaluates its influence on the scheduling performance. A case study based on real PV plant data demonstrates the effectiveness of the proposed policy and shows the influence of parameter variations on the results.

In this paper, the electricity pricing mechanism and payment method of cleaning workers are simplified based on the existing studies. In future research, it is interesting to investigate cleaning scheduling with a more realistic electricity pricing mechanism and payment method. The proposed approach can also be used in other optimal problems with uncertainty or periodic characteristics, such as in periodic piecewise systems combined with some control methods [44], [45].

\section{ACKNOWLEDGMENT}

The authors are grateful to the editor and anonymous referees for their constructive comments, which have significantly improved this paper. The authors also thank Prof. Min Xie and Dr. Xiaolin Wang for their valuable comments and suggestions on this paper.

\section{REFERENCES}

[1] X. Kong, X. Liu, L. Ma, and K. Y. Lee, "Hierarchical distributed model predictive control of standalone wind/solar/battery power system," IEEE Transactions on Systems, Man, and Cybernetics: Systems, vol. 49, no. 8 , pp. 1570-1581, 2019.

[2] T. Ma, H. Yang, and L. Lu, "Solar photovoltaic system modeling and performance prediction," Renewable and Sustainable Energy Reviews, vol. 36, no. 36, pp. 304-315, 2014.

[3] Z. Zhang, C. Dou, B. Zhang, and W. Yue, "Voltage distributed cooperative control considering communication security in photovoltaic power system," IEEE Transactions on Systems, Man, and Cybernetics: Systems, vol. 49, no. 8, pp. 1592-1600, 2019.

[4] Z. Cen and P. Stewart, "Condition parameter estimation for photovoltaic buck converters based on adaptive model observers," IEEE Transactions on Reliability, vol. 66, no. 1, pp. 148-160, 2017.

[5] A. Sayed, M. EL-Shimy, M. El-Metwally, and M. Elshahed, "Impact of subsystems on the overall system availability for the large scale grid-connected photovoltaic systems," Reliability Engineering \& System Safety, vol. 196, p. 106742, 2020.

[6] P. Asef, R. B. Perpina, M. Barzegaran, and A. Lapthorn, "A 3D pareto-based shading analysis on solar photovoltaic system design optimization," IEEE Transactions on Sustainable Energy, vol. 10, no. 2, pp. 843-852, 2018.

[7] M. S. El-Dein, M. Kazerani, and M. Salama, "Optimal photovoltaic array reconfiguration to reduce partial shading losses," IEEE Transactions on Sustainable Energy, vol. 4, no. 1, pp. 145-153, 2012.

[8] R. Appels, B. Lefevre, B. Herteleer, H. Goverde, A. Beerten, R. Paesen, K. De Medts, J. Driesen, and J. Poortmans, "Effect of soiling on photovoltaic modules," Solar Energy, vol. 96, pp. 283-291, 2013.

[9] J. Liu, W. Fang, X. Zhang, and C. Yang, "An improved photovoltaic power forecasting model with the assistance of aerosol index data," IEEE Transactions on Sustainable Energy, vol. 6, no. 2, pp. 434-442, 2015.

[10] S. A. Said, G. Hassan, H. M. Walwil, and N. Al-Aqeeli, "The effect of environmental factors and dust accumulation on photovoltaic modules and dust-accumulation mitigation strategies," Renewable and Sustainable Energy Reviews, vol. 82, pp. 743-760, 2018. 
[11] C.-M. Cheng, S.-L. Tsao, and P.-Y. Lin, "Seeds: a solar-based energyefficient distributed server farm," IEEE Transactions on Systems, Man, and Cybernetics: Systems, vol. 45, no. 1, pp. 143-156, 2014.

[12] A. Azizivahed, A. Arefi, S. G. Jirsaraie, M. Shafie-khah, L. Li, J. Zhang, and J. P. Catalao, "Energy management strategy in dynamic distribution network reconfiguration considering renewable energy resources and storage," IEEE Transactions on Sustainable Energy, vol. 11, no. 2, pp. $662-673,2020$.

[13] A. Faza, "A probabilistic model for estimating the effects of photovoltaic sources on the power systems reliability," Reliability Engineering \& System Safety, vol. 171, pp. 67-77, 2018.

[14] A. M. Pavan, A. Mellit, and D. De Pieri, "The effect of soiling on energy production for large-scale photovoltaic plants," Solar Energy, vol. 85, no. 5, pp. 1128-1136, 2011.

[15] H. Chen, H. Yi, B. Jiang, K. Zhang, and Z. Chen, "Data-driven detection of hot spots in photovoltaic energy systems," IEEE Transactions on Systems, Man, and Cybernetics: Systems, vol. 49, no. 8, pp. 1731-1738, 2019.

[16] E. L. Meyer and E. E. Van Dyk, "Assessing the reliability and degradation of photovoltaic module performance parameters," IEEE Transactions on Reliability, vol. 53, no. 1, pp. 83-92, 2004.

[17] X. Xie, D. Yue, and C. Peng, "Relaxed real-time scheduling stabilization of discrete-time takagi-sugeno fuzzy systems via an alterable-weightsbased ranking switching mechanism," IEEE Transactions on Fuzzy Systems, vol. 26, no. 6, pp. 3808-3819, 2018.

[18] J. A. Rodríguez, M. F. Anjos, P. Côté, and G. Desaulniers, "Accelerating benders decomposition for short-term hydropower maintenance scheduling," European Journal of Operational Research, vol. 289, no. 1, pp. 240-253, 2021.

[19] F. Qiao, Y. Ma, M. Zhou, and Q. Wu, "A novel rescheduling method for dynamic semiconductor manufacturing systems," IEEE Transactions on Systems, Man, and Cybernetics: Systems, vol. 50, no. 5, pp. 1679-1689, 2020.

[20] B. Liu, P. Do, B. Iung, and M. Xie, "Stochastic filtering approach for condition-based maintenance considering sensor degradation," IEEE Transactions on Automation Science and Engineering, vol. 17, no. 1, pp. 177-190, 2020.

[21] X. Li, Y. Jiang, and R. Ruiz, "Methods for scheduling problems considering experience, learning, and forgetting effects," IEEE Transactions on Systems, Man, and Cybernetics: Systems, vol. 48, no. 5, pp. 743-754, 2016.

[22] X. Wang, H. Zhou, A. K. Parlikad, and M. Xie, "Imperfect preventive maintenance policies with unpunctual execution," IEEE Transactions on Reliability, vol. 69, no. 4, pp. 1480-1492, 2020.

[23] R. K. Jones, A. Baras, A. A. Saeeri, A. A. Qahtani, A. O. A. Amoudi, Y. A. Shaya, M. Alodan, and S. A. Al-Hsaien, "Optimized cleaning cost and schedule based on observed soiling conditions for photovoltaic plants in central saudi arabia," IEEE Journal of Photovoltaics, vol. 6, no. 3, pp. 730-738, 2016.

[24] Y. Jiang, L. Lu, and H. Lu, "A novel model to estimate the cleaning frequency for dirty solar photovoltaic (PV) modules in desert environment," Solar Energy, vol. 140, pp. 236-240, 2016.

[25] W. Al-Kouz, S. Al-Dahidi, B. Hammad, and M. Al-Abed, "Modeling and analysis framework for investigating the impact of dust and temperature on PV systems? performance and optimum cleaning frequency," Applied Sciences, vol. 9, no. 7, p. 1397, 2019.

[26] J. W. Zapata, M. A. Perez, S. Kouro, A. Lensu, and A. Suuronen, "Design of a cleaning program for a PV plant based on analysis of energy losses," IEEE Journal of Photovoltaics, vol. 5, no. 6, pp. 1748 $1756,2017$.

[27] B. Hammad, M. Al-Abed, A. Al-Ghandoor, A. Al-Sardeah, and A. AlBashir, "Modeling and analysis of dust and temperature effects on photovoltaic systems' performance and optimal cleaning frequency: Jordan case study," Renewable and Sustainable Energy Reviews, vol. 82, pp. 2218-2234, 2018.

[28] T. Ashley, E. Carrizosa, and E. Fernandez-Cara, "Heliostat field cleaning scheduling for solar power tower plants: A heuristic approach," Applied Energy, vol. 235, pp. 653-660, 2019.

[29] H. T. Ba, M. Cholette, R. Wang, P. Borghesani, L. Ma, and T. Steinberg, "Optimal condition-based cleaning of solar power collectors," Solar Energy, vol. 157, pp. 762-777, 2017.

[30] Z. Wang, Z. Xu, Y. Zhang, and M. Xie, "Optimal cleaning scheduling for photovoltaic systems in the field based on electricity generation and dust deposition forecasting," IEEE Journal of Photovoltaics, vol. 10, no. 4, pp. 1126-1132, 2020.

[31] H. Mo and M. Xie, "A dynamic approach to performance analysis and reliability improvement of control systems with degraded components,"
IEEE Transactions on Systems, Man, and Cybernetics: Systems, vol. 46, no. 10 , pp. 1404-1414, 2016

[32] K. Kashima, H. Aoyama, and Y. Ohta, "Stable process approach to analysis of systems under heavy-tailed noise: Modeling and stochastic linearization," IEEE Transactions on Automatic Control, vol. 64, no. 4, pp. 1344-1357, 2019.

[33] L. Shen, Y. Wang, Q. Zhai, and Y. Tang, "Degradation modeling using stochastic processes with random initial degradation," IEEE Transactions on Reliability, pp. 1-10, 2018.

[34] D. C. Jordan and S. R. Kurtz, "Photovoltaic degradation rates-an analytical review," Progress in Photovoltaics: Research and Applications, vol. 21, no. 1, pp. 12-29, 2013.

[35] M. C. C. de Oliveira, D. A. Cassini, A. S. A. C. Diniz, L. G. Soares, M. M. Viana, L. L. Kazmerski, and V. d. F. C. Lins, "Comparison and analysis of performance and degradation differences of crystalline-Si photovoltaic modules after 15-years of field operation," Solar Energy, vol. 191, pp. 235-250, 2019.

[36] C. Han and H. Lee, "A field-applicable health monitoring method for photovoltaic system," Reliability Engineering \& System Safety, vol. 184, pp. 219-227, 2019.

[37] M. De Felice, M. Petitta, and P. M. Ruti, "Short-term predictability of photovoltaic production over Italy," Renewable Energy, vol. 80, pp. 197-204, 2015.

[38] H. Long, Z. Zhang, and S. Yan, "Analysis of daily solar power prediction with data-driven approaches," Applied Energy, vol. 126, pp. 29-37, 2014.

[39] S. Han, Y.-h. Qiao, J. Yan, Y.-q. Liu, L. Li, and Z. Wang, "Mid-tolong term wind and photovoltaic power generation prediction based on copula function and long short term memory network," Applied Energy, vol. 239, pp. 181-191, 2019.

[40] F. M. Zaihidee, S. Mekhilef, M. Seyedmahmoudian, and B. Horan, "Dust as an unalterable deteriorative factor affecting PV panel's efficiency: Why and how," Renewable and Sustainable Energy Reviews, vol. 65, pp. 1267-1278, 2016

[41] A. A. Hegazy, "Effect of dust accumulation on solar transmittance through glass covers of plate-type collectors," Renewable Energy, vol. 22 , no. 4 , pp. 525-540, 2001.

[42] J. K. Kaldellis and M. Kapsali, "Simulating the dust effect on the energy performance of photovoltaic generators based on experimental measurements," Energy, vol. 36, no. 8, pp. 5154-5161, 2011.

[43] S. Pelland, J. Remund, J. Kleissl, T. Oozeki, and K. De Brabandere, "Photovoltaic and solar forecasting: state of the art," IEA PVPS, Task, vol. 14, pp. 1-36, 2013.

[44] X. Xie, J. Lam, C. Fan, X. Wang, and K.-W. Kwok, "Energy-to-peak output tracking control of actuator saturated periodic piecewise timevarying systems with nonlinear perturbations," IEEE Transactions on Systems, Man, and Cybernetics: Systems, 2021.

[45] X. Xie, J. Lam, and K.-W. Kwok, "A novel scheme of nonfragile controller design for periodic piecewise 1tv systems," IEEE Transactions on Industrial Electronics, vol. 67, no. 12, pp. 10766-10775, 2020. 